\title{
Chemical Kinetics of Asphaltene Pyrolysis
}

\section{Paolo Guida, $*, \dagger$ Eleonora Colombo, $¥$ Elia Colleoni, $¥$ Saumitra Saxena, $†$ Alessio Frassoldati, $\ddagger$ William L. Roberts, $\dagger$ and Tiziano Faravelli}

${ }^{\dagger}$ Clean Combustion Research Center, King Abdullah University of Science and Technology,

Thuwal, 23955-6900, Saudi Arabia

${ }^{\ddagger}$ Department of Chemistry, Materials and Chemical Engineering "G. Natta", Piazza L. Da Vinci 32, 20133 Milano, Italy

\section{*Corresponding author e-mail: paolo.guida@,kaust.edu.sa}

Elemental composition data relative to asphaltene collected from literature (empty spaces refer to asphaltenes from the source declared above):

\begin{tabular}{|c|l|c|c|c|c|c|c|}
\hline & Asphaltenes & $\mathbf{C}$ wt\% & $\mathbf{H}$ wt\% & $\mathbf{N}$ wt\% & $\mathbf{S ~ w t \%}$ & $\mathbf{O} \mathbf{w t} \%$ & $\mathbf{H} \mathbf{C}$ \\
\hline 1 & $\begin{array}{l}\text { Turkish crude oil - } \\
\text { Batiraman [1] }\end{array}$ & 79.23 & 7.23 & 1.66 & 8.83 & 3.05 & 1.10 \\
\hline 2 & $\begin{array}{l}\text { Liaohe vacuum } \\
\text { residue [2] }\end{array}$ & 86.13 & 7.76 & 2.21 & 0.71 & 3.19 & 1.08 \\
\hline 3 & $\begin{array}{l}\text { Venezuelan } \\
\text { vacuum residue } \\
\text { [2] }\end{array}$ & 81.96 & 7.08 & 1.26 & 6.52 & 3.18 & 1.04 \\
\hline 4 & $\begin{array}{l}\text { Indonesian oil } \\
\text { sand bitumen [3] }\end{array}$ & 78.28 & 8.08 & 0.88 & 9.51 & 3.26 & 1.24 \\
\hline 5 & $\begin{array}{l}\text { Iranian vacuum } \\
\text { residue [4] }\end{array}$ & 84.69 & 7.61 & 1.31 & 6.40 & 0.00 & 1.08 \\
\hline 6 & Alberta [5] & 82.68 & 8.34 & 1.20 & 7.75 & 0.03 & 1.21 \\
\hline 7 & Cold Lake [6] & 81.11 & 8.41 & 1.25 & 7.59 & 1.64 & 1.24 \\
\hline 8 & Maya crude oil [7] & 84.71 & 7.50 & 1.25 & 5.77 & 0.76 & 1.06 \\
\hline 9 & HFO [8] & 83.49 & 8.03 & 0.75 & 7.07 & 0.66 & 1.15 \\
\hline 10 & $\begin{array}{l}\text { Arabian light } \\
\text { crude oil 5 [9] }\end{array}$ & 82.37 & 7.85 & 0.78 & 6.50 & 2.50 & 1.14 \\
\hline 11 & $\begin{array}{l}\text { Arabian heavy } \\
\text { crude oil 5 }\end{array}$ & 82.38 & 7.65 & 0.85 & 7.43 & 1.69 & 1.11 \\
\hline 12 & $\begin{array}{l}\text { Arabian heavy } \\
\text { crude oil 7 }\end{array}$ & 82.15 & 7.40 & 0.96 & 7.61 & 1.88 & 1.08 \\
\hline 13 & $\begin{array}{l}\text { Arabian heavy } \\
\text { crude oil 10 }\end{array}$ & 81.39 & 7.30 & 1.31 & 7.79 & 2.21 & 1.08 \\
\hline 14 & $\begin{array}{l}\text { Wilmington } \\
\text { California [10] }\end{array}$ & 83.68 & 8.57 & 2.18 & 2.52 & 2.80 & 1.23 \\
\hline
\end{tabular}




\begin{tabular}{|c|l|c|c|c|c|c|c|}
\hline 15 & $\begin{array}{l}\text { Canada npentane } \\
{[11]}\end{array}$ & 79.50 & 8.00 & 1.20 & 7.50 & 3.80 & 1.21 \\
\hline 16 & Canada nheptane & 78.40 & 7.60 & 1.40 & 8.00 & 4.60 & 1.16 \\
\hline 17 & Iran n-pentane & 83.80 & 7.50 & 1.40 & 5.00 & 2.30 & 1.07 \\
\hline 18 & Iran n-heptane & 84.20 & 7.00 & 1.60 & 5.80 & 1.40 & 1.00 \\
\hline 19 & Iraq n-pentane & 81.70 & 7.90 & 0.80 & 8.50 & 1.10 & 1.16 \\
\hline 20 & Iraq n-heptane & 80.70 & 7.10 & 0.90 & 9.80 & 1.50 & 1.06 \\
\hline 21 & Kuwait n-pentane & 82.40 & 7.90 & 0.90 & 7.40 & 1.40 & 1.15 \\
\hline 22 & Kuwait n-heptane & 82.00 & 7.30 & 1.00 & 7.80 & 1.90 & 1.07 \\
\hline 23 & Athabasca A [12] & 80.50 & 8.10 & 1.10 & 1.90 & 7.40 & 1.21 \\
\hline 24 & Athabasca B & 81.30 & 8.20 & 1.20 & 1.90 & 3.40 & 1.21 \\
\hline 25 & $\begin{array}{l}\text { Bachequero } \\
\text { Venezuela }\end{array}$ & 85.50 & 7.70 & 2.00 & 3.00 & 2.40 & 1.08 \\
\hline 26 & $\begin{array}{l}\text { Baxterville A } \\
\text { Missisipi }\end{array}$ & 84.50 & 7.20 & 1.40 & 0.50 & 3.50 & 1.02 \\
\hline 27 & Belridge & 83.80 & 8.30 & 2.60 & 2.20 & 0.50 & 1.19 \\
\hline
\end{tabular}

\begin{tabular}{|c|l|c|c|c|c|c|c|}
\hline 28 & $\begin{array}{l}\text { Boscan A } \\
\text { Venezuela }\end{array}$ & 80.50 & 7.70 & 1.90 & 2.80 & 6.20 & 1.15 \\
\hline 29 & $\begin{array}{l}\text { Boscan B } \\
\text { Venezuela }\end{array}$ & 80.80 & 7.80 & 2.20 & 2.40 & 7.50 & 1.16 \\
\hline 30 & Libya & 85.20 & 8.90 & 0.70 & 0.67 & 0.50 & 1.25 \\
\hline 31 & $\begin{array}{l}\text { Melones } \\
\text { Venezuela }\end{array}$ & 83.40 & 8.20 & 2.00 & 0.80 & 6.10 & 1.18 \\
\hline 32 & Rozel Point Utah & 71.80 & 8.40 & 1.40 & 3.40 & 15.30 & 1.40 \\
\hline 33 & $\begin{array}{l}\text { Santiago } \\
\text { California }\end{array}$ & 84.40 & 8.60 & 2.00 & 2.20 & 1.80 & 1.22 \\
\hline 34 & $\begin{array}{l}\text { Yorba Linda } \\
\text { California }\end{array}$ & 82.20 & 8.30 & 1.40 & 2.30 & 2.00 & 1.21 \\
\hline 35 & $\begin{array}{l}n \text {-pentane } \\
\text { Venezuelan } \\
\text { asphaltenes }[13]\end{array}$ & 83.20 & 8.00 & 2.70 & 5.10 & 1.40 & 1.15 \\
\hline 36 & $\begin{array}{l}n \text {-heptane } \\
\text { Venezuelan } \\
\text { asphaltenes }\end{array}$ & 82.70 & 7.50 & 1.80 & 4.90 & 1.50 & 1.09 \\
\hline 37 & $\begin{array}{l}n \text {-nonane } \\
\text { Venezuelan } \\
\text { asphaltenes }\end{array}$ & 82.60 & 7.70 & 3.00 & 4.90 & 1.80 & 1.12 \\
\hline 38 & $\begin{array}{l}\text { Maya n-pentane } \\
\text { Mexican }\end{array}$ & 81.23 & 8.11 & 1.32 & 8.25 & 0.97 & 1.20 \\
\hline Maya n-heptane & 81.62 & 7.26 & 1.46 & 8.46 & 1.02 & 1.07 \\
\hline
\end{tabular}




\begin{tabular}{|c|l|c|c|c|c|c|c|}
\hline 40 & $\begin{array}{l}\text { Isthmus npentane } \\
\text { Mexican }\end{array}$ & 83.90 & 8.00 & 1.33 & 6.06 & 0.71 & 1.14 \\
\hline 41 & Isthmus nheptane & 83.99 & 7.30 & 1.35 & 6.48 & 0.79 & 1.04 \\
\hline 42 & $\begin{array}{l}\text { Olmeca npentane } \\
\text { Mexican }\end{array}$ & 86.94 & 7.91 & 1.33 & 3.20 & 0.62 & 1.09 \\
\hline 43 & Olmeca nheptane & 87.16 & 7.38 & 1.34 & 3.48 & 0.64 & 1.02 \\
\hline 44 & $\begin{array}{l}\text { Hassi-Messaoud } \\
\text { Algeria }\end{array}$ & 86.32 & 13.18 & 0.03 & 0.15 & 0.32 & 1.83 \\
\hline 45 & - & 90.12 & 9.02 & 0.03 & 0.90 & 0.00 & 1.20 \\
\hline 46 & - & 90.22 & 7.60 & 0.49 & 0.92 & 0.73 & 1.01 \\
\hline 47 & - & 86.08 & 8.57 & 0.64 & 1.25 & 3.46 & 1.19 \\
\hline 48 & Laghouat Algeria & 88.61 & 8.32 & 0.05 & 0.40 & 2.62 & 1.13 \\
\hline 49 & - & 85.90 & 8.70 & 0.75 & 1.60 & 3.05 & 1.22 \\
\hline 50 & Iraq & 80.40 & 8.07 & 0.34 & 8.21 & 2.98 & 1.20 \\
\hline 51 & - & 79.99 & 7.26 & 0.71 & 5.46 & 6.58 & 1.09 \\
\hline 52 & - & 78.37 & 8.83 & 0.34 & 6.71 & 5.75 & 1.35 \\
\hline 53 & Boscan & 85.10 & 12.70 & 0.10 & 0.80 & 1.30 & 1.79 \\
\hline 54 & $\begin{array}{l}\text { Scheibengard } \\
\text { France }\end{array}$ & 87.60 & 8.62 & 0.06 & 0.72 & 3.00 & 1.18 \\
\hline 55 & - & 88.02 & 8.30 & 0.08 & 0.50 & 3.10 & 1.13 \\
\hline 56 & Shale bitumen & 74.35 & 8.86 & 2.71 & 1.80 & 10.32 & 1.43 \\
\hline
\end{tabular}

\begin{tabular}{|c|l|c|c|c|c|c|c|}
\hline 57 & Lake sediment & 72.47 & 9.16 & 2.03 & 0.31 & 16.03 & 1.52 \\
\hline 58 & $\begin{array}{l}\text { Sporopollenin } \\
\text { (kerogen of type } \\
\text { II) degradation }\end{array}$ & 83.50 & 7.72 & 0.10 & 0.00 & 8.68 & 1.11 \\
\hline 59 & Volga-Ural & 79.05 & 6.49 & 1.52 & 1.45 & 11.49 & 0.99 \\
\hline 60 & & 76.38 & 7.33 & 2.78 & 3.42 & 10.09 & 1.15 \\
\hline 61 & & 74.87 & 6.79 & 1.37 & 2.76 & 14.21 & 1.09 \\
\hline 62 & Fecocourt & 77.36 & 8.53 & 0.86 & 4.83 & 8.42 & 1.32 \\
\hline 63 & Der & 80.17 & 8.80 & 1.63 & 4.96 & 4.44 & 1.32 \\
\hline 64 & Essises & 85.45 & 7.59 & 1.71 & 1.15 & 4.10 & 1.07 \\
\hline 65 & Ultra-Bulak & 87.10 & 8.05 & 0.05 & 4.50 & 0.30 & 1.11 \\
\hline 66 & Arlanskoe & 83.70 & 9.70 & 1.12 & 4.36 & 1.12 & 1.39 \\
\hline 67 & Krasnoborsk & 87.93 & 7.85 & 0.80 & 0.82 & 2.60 & 1.07 \\
\hline 68 & Volga-Ural & 87.17 & 8.30 & 1.10 & 1.68 & 1.75 & 1.14 \\
\hline 69 & $\begin{array}{l}\text { Sedimentary rock } \\
\text { bitumen }\end{array}$ & 77.80 & 7.50 & 1.47 & 5.67 & 7.56 & 1.16 \\
\hline 70 & $\begin{array}{l}\text { Scheiben-hard } \\
\text { France }\end{array}$ & 87.88 & 7.71 & 0.20 & 0.70 & 3.51 & 1.05 \\
\hline
\end{tabular}




\begin{tabular}{|c|l|c|c|c|c|c|c|}
\hline 71 & Hassi-Messaoud & 88.20 & 6.50 & 0.50 & 0.80 & 4.00 & 0.88 \\
\hline 72 & Boscan & 81.10 & 7.80 & 0.20 & 6.70 & 4.20 & 1.15 \\
\hline 73 & Tuimazy Russia & 83.00 & 7.60 & 0.23 & 5.30 & 3.87 & 1.10 \\
\hline 74 & - & 83.10 & 8.30 & 0.15 & 4.80 & 3.65 & 1.20 \\
\hline 75 & Bachaquero & 85.00 & 5.80 & 1.40 & 4.60 & 1.50 & 0.82 \\
\hline 76 & $\begin{array}{l}\text { Green River shale } \\
\text { Colorado,Wyomin } \\
\text { g,Utah }\end{array}$ & 81.25 & 8.24 & 4.31 & 0.81 & 3.04 & 1.22 \\
\hline 77 & $\begin{array}{l}\text { Kentucky (volatile } \\
\text { coal) }\end{array}$ & 85.91 & 6.85 & 1.67 & 1.46 & 4.11 & 0.96 \\
\hline 78 & - & 85.30 & 6.44 & 1.75 & 0.67 & 5.85 & 0.91 \\
\hline 79 & $\begin{array}{l}\text { Liddell Australia } \\
\text { (medium-volatile } \\
\text { bitumen coal) }\end{array}$ & 85.80 & 7.10 & 1.52 & 0.18 & 5.40 & 0.99 \\
\hline 80 & - & 85.70 & 6.50 & 1.74 & 0.06 & 6.00 & 0.91 \\
\hline 81 & $\begin{array}{l}\text { Cold Lake } \\
\text { nonbasic Canada } \\
{[14]}\end{array}$ & 80.70 & 7.80 & 1.10 & 7.80 & 0.00 & 1.16 \\
\hline 82 & $\begin{array}{l}\text { Cold Lake basic } \\
\text { Canada }\end{array}$ & 80.60 & 7.60 & 1.40 & 7.60 & 0.00 & 1.13 \\
\hline 83 & $\begin{array}{l}\text { Wyodak nonbasic } \\
\text { Canada }\end{array}$ & 87.70 & 6.40 & 0.60 & 0.60 & 3.90 & 0.88 \\
\hline 84 & $\begin{array}{l}\text { Wyodak basic } \\
\text { Canada }\end{array}$ & 84.10 & 6.30 & 3.20 & 0.60 & 5.50 & 0.90 \\
\hline Kuwait [15] & 83.01 & 9.43 & 0.81 & 3.37 & 3.38 & 1.36 \\
\hline $\begin{array}{l}\text { Monagal Venezuela } \\
\text { [16] }\end{array}$ & 85.50 & 6.90 & 1.73 & 3.40 & 2.50 & 0.97 \\
\hline
\end{tabular}

\begin{tabular}{|c|l|c|c|c|c|c|c|}
\hline 87 & $\begin{array}{l}\text { DM 153 } \\
\text { Venezuela } \\
\text { Maracaibo Lake }\end{array}$ & 81.05 & 8.17 & 1.64 & 6.60 & 2.00 & 1.21 \\
\hline 89 & $\begin{array}{l}\text { DM 115 } \\
\text { Venezuela } \\
\text { Maracaibo Lake }\end{array}$ & 80.23 & 7.73 & 1.44 & 6.30 & 4.40 & 1.16 \\
\hline $\begin{array}{l}\text { VLA 711 } \\
\text { Venezuela } \\
\text { Maracaibo Lake }\end{array}$ & $\begin{array}{l}\text { Hamaca } \\
\text { Venezuela } \\
\text { Orinoco }\end{array}$ & 83.94 & 7.53 & 1.23 & 4.60 & 2.00 & 1.08 \\
\hline
\end{tabular}




\begin{tabular}{|c|c|c|c|c|c|c|c|}
\hline 91 & $\begin{array}{l}\text { Cerro Negro } \\
\text { Venezuela } \\
\text { Orinoco }\end{array}$ & 81.23 & 7.72 & 2.13 & 5.50 & 3.50 & 1.14 \\
\hline 92 & $\begin{array}{l}\text { Wilmington } \\
\text { california [17] }\end{array}$ & 83.68 & 8.57 & 2.18 & 2.52 & 2.80 & 1.23 \\
\hline 93 & Campana [18] & 87.60 & 8.20 & 1.50 & 0.50 & 2.20 & 1.12 \\
\hline 94 & $\begin{array}{l}\text { Heavy Canadian (Cold } \\
\text { Lake) }\end{array}$ & 84.70 & 7.90 & 1.20 & 4.50 & 1.60 & 1.12 \\
\hline 95 & $\begin{array}{l}\text { LloydminsterWainwright } \\
\text { Alberta }\end{array}$ & 80.00 & 8.00 & 1.30 & 7.90 & 2.70 & 1.20 \\
\hline 96 & Maya & 82.30 & 7.90 & 1.20 & 6.60 & 1.80 & 1.15 \\
\hline 97 & Mid-continent US & 84.90 & 8.60 & 1.00 & 3.80 & 1.60 & 1.22 \\
\hline 98 & $\begin{array}{l}\text { San Joaquin } \\
\text { Valley California }\end{array}$ & 84.50 & 8.30 & 2.60 & 2.30 & 2.30 & 1.18 \\
\hline 99 & $\begin{array}{l}\text { Athabasca industrial } n- \\
\text { C5 } \\
{[19]}\end{array}$ & 81.60 & 8.50 & 1.40 & 7.70 & 0.80 & 1.25 \\
\hline 100 & Athabasca $\mathrm{n}-\mathrm{C7}$ & 81.50 & 7.60 & 1.60 & 8.00 & 1.30 & 1.12 \\
\hline 101 & Cold Lake n-C7 & 77.60 & 7.60 & 1.60 & 8.30 & 4.90 & 1.18 \\
\hline 102 & Gudao n-C7 China & 81.60 & 8.40 & 2.10 & 6.30 & 1.60 & 1.24 \\
\hline 103 & Maya n-C7 & 82.10 & 7.70 & 1.80 & 7.50 & 0.90 & 1.13 \\
\hline 104 & $\begin{array}{l}\text { Safaniya n-C7 Saudi } \\
\text { Arabia }\end{array}$ & 82.00 & 7.70 & 1.50 & 7.90 & 0.90 & 1.13 \\
\hline 105 & Venezuelan n-C7 & 81.80 & 8.00 & 2.30 & 6.10 & 1.80 & 1.17 \\
\hline 106 & A47T [20] & 76.50 & 7.46 & 1.16 & 6.41 & 8.45 & 1.17 \\
\hline 107 & A185T & 76.66 & 7.39 & 1.22 & 6.42 & 8.30 & 1.16 \\
\hline 108 & A321T & 78.67 & 7.73 & 1.23 & 6.91 & 5.46 & 1.18 \\
\hline 109 & A366T & 76.85 & 7.44 & 1.26 & 6.57 & 7.88 & 1.16 \\
\hline 110 & Gela [21] & 79.70 & 6.90 & 1.10 & 10.80 & 1.50 & 1.04 \\
\hline 111 & Belayim & 83.90 & 8.10 & 1.90 & 5.30 & 0.80 & 1.16 \\
\hline 112 & Safaniya & 82.40 & 7.70 & 1.00 & 7.70 & 1.20 & 1.12 \\
\hline 113 & Arabian Light & 84.10 & 7.00 & 1.00 & 7.10 & 0.70 & 1.00 \\
\hline 114 & Brent & 86.90 & 7.40 & 1.10 & 2.10 & 2.60 & 1.02 \\
\hline 115 & Gaggiano & 82.80 & 6.80 & 1.40 & 2.90 & 6.20 & 0.99 \\
\hline
\end{tabular}

\begin{tabular}{|c|l|c|c|c|c|c|c|}
\hline 116 & Villa fortuna & 90.30 & 6.10 & 1.00 & 1.90 & 0.80 & 0.81 \\
\hline 117 & $\begin{array}{l}\text { Arabian } \\
\text { Asphaltenes [22] }\end{array}$ & 84.89 & 8.49 & 0.62 & 4.98 & 1.02 & 1.20 \\
\hline 118 & $\begin{array}{l}\text { Arabian } \\
\text { Asphaltenes }\end{array}$ & 82.84 & 8.21 & 0.71 & 4.98 & 3.26 & 1.19 \\
\hline
\end{tabular}




\begin{tabular}{|c|c|c|c|c|c|c|c|}
\hline 119 & $\begin{array}{l}\text { Arabian Light } \\
\text { Vacuum Residue } \\
{[23]}\end{array}$ & 84.06 & 7.59 & 0.75 & 6.17 & 1.43 & 1.08 \\
\hline 120 & $\begin{array}{l}\text { Vacuum residue } \\
{[24]}\end{array}$ & 81.93 & 7.94 & 1.15 & 7.50 & 1.55 & 1.16 \\
\hline 121 & $\begin{array}{l}\text { Tahe heavy oil } \\
\text { [25] }\end{array}$ & 83.96 & 7.58 & 1.40 & 4.20 & 2.86 & 1.08 \\
\hline 122 & Liaohe heavy oil & 85.95 & 7.22 & 1.95 & 1.23 & 3.65 & 1.01 \\
\hline 123 & Pembina [12] & 87.91 & 8.29 & 1.00 & 0.95 & 1.84 & 1.13 \\
\hline 124 & Pembina & 87.80 & 8.03 & 1.20 & 1.00 & 1.97 & 1.10 \\
\hline 125 & Ferrier & 88.95 & 8.23 & 1.14 & 1.47 & 1.20 & 1.11 \\
\hline 126 & Pembina & 88.42 & 8.01 & 1.30 & 1.01 & 1.27 & 1.09 \\
\hline 127 & Gilby & 84.14 & 10.66 & 1.11 & 1.06 & 3.03 & 1.52 \\
\hline 128 & Crossfield & 89.39 & 7.63 & 1.12 & 0.51 & 1.34 & 1.02 \\
\hline 129 & Barons & 89.28 & 7.49 & 1.17 & 0.92 & 1.13 & 1.01 \\
\hline 130 & Crossfield & 88.49 & 8.21 & 1.61 & 0.31 & 1.38 & 1.11 \\
\hline 131 & Cyn-Pem & 86.84 & 10.21 & 1.29 & 0.56 & 1.10 & 1.41 \\
\hline 132 & Carrington & 85.14 & 11.10 & 0.66 & 0.59 & 2.51 & 1.56 \\
\hline 133 & Giroux Lake & 89.22 & 7.64 & 0.93 & 0.60 & 1.61 & 1.03 \\
\hline 134 & Legal & 89.01 & 7.66 & 1.25 & 0.98 & 1.11 & 1.03 \\
\hline 135 & Joarcam & 87.08 & 7.79 & 1.16 & 0.99 & 2.98 & 1.07 \\
\hline 136 & Crossfield & 89.40 & 8.07 & 0.99 & 0.18 & 1.36 & 1.08 \\
\hline 137 & Provost & 88.13 & 7.96 & 1.14 & 1.08 & 1.43 & 1.08 \\
\hline 138 & Cessford & 84.70 & 7.78 & 1.23 & 5.20 & 1.08 & 1.10 \\
\hline 139 & Joarcam & 85.48 & 7.88 & 1.44 & 0.89 & 4.31 & 1.11 \\
\hline 140 & Willesden Green & 87.02 & 7.11 & 1.43 & 3.41 & 1.03 & 0.98 \\
\hline 141 & Medicine River & 86.29 & 7.54 & 1.16 & 4.00 & 1.02 & 1.05 \\
\hline 142 & Kaybob & 86.01 & 7.34 & 0.89 & 5.06 & 0.70 & 1.02 \\
\hline 143 & Niton & 84.12 & 8.35 & 0.73 & 2.68 & 4.12 & 1.19 \\
\hline 144 & Pembina & 85.72 & 7.45 & 1.31 & 4.48 & 1.04 & 1.04 \\
\hline 145 & Gilby Alexander & 82.31 & 7.72 & 2.33 & 6.37 & 1.27 & 1.13 \\
\hline 146 & Gilby & 86.05 & 6.65 & 1.15 & 5.63 & 0.53 & 0.93 \\
\hline 147 & Medicine River & 86.10 & 6.90 & 1.11 & 5.33 & 0.56 & 0.96 \\
\hline 148 & Medicine River & 84.30 & 7.43 & 1.34 & 4.87 & 2.06 & 1.06 \\
\hline 149 & $\begin{array}{l}\text { Boundary Lake } \\
\text { South }\end{array}$ & 86.26 & 7.89 & 1.49 & 3.52 & 0.83 & 1.10 \\
\hline 150 & Worsley & 82.90 & 7.68 & 1.36 & 6.92 & 1.13 & 1.11 \\
\hline 151 & $\begin{array}{l}\text { Sturgeon Lake } \\
\text { South }\end{array}$ & 84.08 & 7.41 & 1.33 & 6.12 & 1.05 & 1.06 \\
\hline 152 & Eaglesham & 87.62 & 7.49 & 1.14 & 2.09 & 1.65 & 1.03 \\
\hline 153 & Crossfield & 87.53 & 8.05 & 0.07 & 2.76 & 0.95 & 1.10 \\
\hline
\end{tabular}




\begin{tabular}{|c|c|c|c|c|c|c|c|}
\hline 154 & Del Sonita & 84.87 & 8.41 & 0.63 & 4.23 & 1.86 & 1.19 \\
\hline 155 & Sylvan Lake & 86.31 & 7.50 & 1.36 & 3.37 & 1.46 & 1.04 \\
\hline 156 & Twining & 86.29 & 7.11 & 0.99 & 4.97 & 0.64 & 0.99 \\
\hline 157 & Cherhill & 82.32 & 8.06 & 1.69 & 6.53 & 1.40 & 1.17 \\
\hline 158 & Swalwell & 85.64 & 7.55 & 1.11 & 3.83 & 1.87 & 1.06 \\
\hline 159 & Princess & 84.47 & 7.62 & 1.28 & 5.50 & 1.13 & 1.08 \\
\hline 160 & Leduc-Woodbend & 85.57 & 7.86 & 1.42 & 1.29 & 3.86 & 1.10 \\
\hline 161 & Maimo & 85.65 & 7.73 & 1.40 & 4.21 & 1.02 & 1.08 \\
\hline 162 & Nix & 82.85 & 9.14 & 2.04 & 1.53 & 4.41 & 1.32 \\
\hline 163 & Youngstown & 85.29 & 7.44 & 1.88 & 4.57 & 0.82 & 1.05 \\
\hline 164 & Joffre & 85.72 & 7.90 & 0.83 & 2.59 & 2.96 & 1.11 \\
\hline 165 & Fenn-Big Valley & 86.21 & 8.07 & 1.56 & 3.64 & 0.52 & 1.12 \\
\hline 166 & Youngstown & 85.74 & 7.23 & 1.15 & 4.92 & 0.96 & 1.01 \\
\hline 167 & Bonnie Glen & 89.09 & 7.60 & 0.73 & 1.22 & 1.37 & 1.02 \\
\hline 168 & Westerose & 86.97 & 7.75 & 1.23 & 2.00 & 2.04 & 1.07 \\
\hline 169 & Maimo & 87.05 & 7.62 & 1.59 & 3.29 & 0.45 & 1.05 \\
\hline 170 & Erskine & 84.60 & 6.46 & 1.24 & 6.78 & 0.92 & 0.92 \\
\hline 171 & Loon & 89.53 & 7.43 & 1.04 & 1.21 & 0.79 & 1.00 \\
\hline 172 & Loon & 87.07 & 9.45 & 1.02 & 0.76 & 1.70 & 1.30 \\
\hline 173 & Swan Hills & 89.70 & 6.84 & 0.92 & 0.90 & 1.64 & 0.92 \\
\hline 174 & Goose River & 89.71 & 7.00 & 1.02 & 0.75 & 1.52 & 0.94 \\
\hline 175 & Zama & 86.46 & 8.52 & 0.91 & 3.57 & 0.54 & 1.18 \\
\hline 176 & Zama & 84.49 & 7.45 & 2.37 & 4.46 & 1.23 & 1.06 \\
\hline 177 & Zama & 86.83 & 7.86 & 0.92 & 3.82 & 0.56 & 1.09 \\
\hline 178 & Zama & 85.71 & 7.81 & 1.36 & 5.82 & 1.30 & 1.09 \\
\hline 179 & Nipisi & 89.59 & 7.49 & 1.00 & 1.29 & 0.63 & 1.00 \\
\hline 180 & Zama & 86.40 & 9.03 & 1.66 & 0.78 & 2.13 & 1.25 \\
\hline 181 & Nipisi & 89.62 & 7.34 & 1.02 & 1.18 & 0.84 & 0.98 \\
\hline 182 & Gilwood & 87.94 & 7.61 & 2.21 & 0.47 & 1.77 & 1.04 \\
\hline 183 & Loon & 89.79 & 7.40 & 0.96 & 1.22 & 0.62 & 0.99 \\
\hline 184 & Red Earth & 86.50 & 7.74 & 1.30 & 1.41 & 3.05 & 1.07 \\
\hline 185 & $\begin{array}{l}\text { Woydminster } \\
\text { (Sparky J) }\end{array}$ & 81.94 & 7.96 & 1.00 & 7.96 & 1.14 & 1.17 \\
\hline 186 & $\begin{array}{l}\text { Wildmere } \\
\text { (Sparky) }\end{array}$ & 82.18 & 8.18 & 1.59 & 7.61 & 0.44 & 1.19 \\
\hline 187 & $\begin{array}{l}\text { Lloydminster } \\
\text { (Blairmore) }\end{array}$ & 81.91 & 8.11 & 1.18 & 7.80 & 1.00 & 1.19 \\
\hline 188 & $\begin{array}{l}\text { Chauvin } \\
\text { (Mannville A) }\end{array}$ & 82.81 & 7.87 & 1.22 & 6.72 & 1.38 & 1.14 \\
\hline 189 & Inland (Colony) & 80.42 & 7.81 & 2.59 & 7.19 & 1.99 & 1.17 \\
\hline 190 & $\begin{array}{l}\text { South Taber } \\
\text { (Mannville A) }\end{array}$ & 82.73 & 7.75 & 2.82 & 5.75 & 0.95 & 1.12 \\
\hline
\end{tabular}




\begin{tabular}{|c|l|c|c|c|c|c|c|}
\hline 191 & $\begin{array}{l}\text { Alderson (Lower } \\
\text { Mannville B) }\end{array}$ & 82.79 & 7.52 & 2.14 & 5.97 & 1.58 & 1.09 \\
\hline 192 & $\begin{array}{l}\text { Bantry (Mannville } \\
\text { A) }\end{array}$ & 83.30 & 7.79 & 1.48 & 5.55 & 1.88 & 1.12 \\
\hline 193 & $\begin{array}{l}\text { Chauvin South } \\
\text { (Sparky C) }\end{array}$ & 82.64 & 8.21 & 1.36 & 6.23 & 1.56 & 1.19 \\
\hline 194 & Athabasca & 80.31 & 8.37 & 1.09 & 7.77 & 2.45 & 1.25 \\
\hline 195 & Athabasca & 80.47 & 8.45 & 1.25 & 7.95 & 1.88 & 1.26 \\
\hline 196 & Athabasca & 82.63 & 8.35 & 1.43 & 5.75 & 1.85 & 1.21 \\
\hline 197 & Athabasca & 80.62 & 8.31 & 1.24 & 8.21 & 1.61 & 1.24 \\
\hline 198 & Wabasca & 81.00 & 8.32 & 0.99 & 7.57 & 2.11 & 1.23 \\
\hline 199 & Wabasca & 80.13 & 8.29 & 1.58 & 8.09 & 1.91 & 1.24 \\
\hline 200 & Wabasca & 81.01 & 8.13 & 0.94 & 7.70 & 2.21 & 1.20 \\
\hline 201 & Wabasca & 80.06 & 8.23 & 1.20 & 7.93 & 2.58 & 1.23 \\
\hline 202 & Cold Lake & 83.33 & 8.41 & 1.23 & 5.39 & 1.64 & 1.21 \\
\hline 203 & Cold Lake & 80.76 & 8.25 & 1.14 & 7.21 & 2.61 & 1.23 \\
\hline 204 & Cold Lake & 81.04 & 8.35 & 1.24 & 7.14 & 2.24 & 1.24 \\
\hline 205 & Peace River & 81.04 & 8.33 & 1.07 & 7.73 & 1.83 & 1.23 \\
\hline 206 & Conrad (Ellir) & 66.61 & 7.64 & 1.32 & 3.47 & 0.96 & 1.38 \\
\hline 207 & Glenevis (Banff) & 83.18 & 7.73 & 1.01 & 6.71 & 1.37 & 1.12 \\
\hline 208 & Glenevis (Banff) & 82.91 & 8.23 & 1.50 & 6.84 & 0.52 & 1.19 \\
\hline & & & & & & & \\
\hline
\end{tabular}


[1] T. Ren, Y. Wang, and G. Zhang, "Influence of the properties of resins on the interactions between asphaltenes and resins," Pet. Sci. Technol., vol. 35, no. 14, pp. 1481-1486, 2017.

[2] K. Chen, H. Liu, A. Guo, D. Ge, and Z. Wang, "Study of the thermal performance and interaction of petroleum residue fractions during the coking process," Energy and Fuels, vol. 26, no. 10, pp. 6343-6351, 2012.

[3] Y. Che, Z. Yang, Y. Qiao, J. Zhang, and Y. Tian, "Study on pyrolysis characteristics and kinetics of vacuum residue and its eight group-fractions by TG-FTIR," Thermochim. Acta, vol. 669, no. June, pp. 149-155, 2018.

[4] J. Zhang, Y. Tian, Y. Qiao, C. Yang, and H. Shan, "Structure and Reactivity of Iranian Vacuum Residue and Its Eight Group-Fractions," Energy and Fuels, vol. 31, no. 8, pp. 8072-8086, 2017.

[5] N. Mahapatra, "Pyrolysis of asphaltenes in an atmospheric entrained flow reactor: A study on gasification reactivity and properties of chars," 2014.

[6] K. C. Khulbe, A. K. Sachdev, R. S. Mann, and S. Davis, "TGA studies of asphaltenes derived from cold-lake (Canada) bitumen," Fuel Process. Technol., vol. 8, no. 3, pp. 259-266, 1984.

[7] J. Douda, M. E. Llanos, R. Alvarez, C. L. Franco, and J. A. M. De La Fuente, "Pyrolysis applied to the study of a Maya asphaltene," J. Anal. Appl. Pyrolysis, vol. 71, no. 2, pp. 601-612, 2004.

[8] V. Garaniya, D. McWilliam, L. Goldsworthy, and M. Ghiji, "Extensive chemical characterization of a heavy fuel oil," Fuel, vol. 227, no. April, pp. 67-78, 2018.

[9] M. F. Ali and A. Bukhari, "Thermal decomposition of Saudi crude oil asphaltenes," Fuel Sci. Technol. Int., vol. 9, no. 4, 1991.

[10] J. F. McKAY, P. J. AMEND, T. E. COGSWELL, P. M. HARNSBERGER, R. B. ERICKSON, and D. R. LATHAM, "Petroleum Asphaltenes: Chemistry and Composition," pp. 128-142, 2009.

[11] J. G. SPEIGHT and S. E. MOSCHOPEDIS, "On the Molecular Nature of Petroleum Asphaltenes," pp. 1-15, 2009.

[12] T. F. Yen and G. V Chilingar, "Asphaltenes and asphalts," vol. 1, p. 459, 1994.

[13] A. Pina, P. Mougin, and E. Béhar, "Characterisation of asphaltenes and modelling of flocculation - State of the art," Oil Gas Sci. Technol., vol. 61, no. 3, pp. 319343, 2006.

[14] M. M. Ramirez-corredores, The Science and Technology of Unconventional Oils. Finding Refining Opportunities. 2017.

[15] Y.-J. Liu and Z.-F. Li, "Structural Characterisation of Asphaltenes during Residue Hydrotreatment with Light Cycle Oil as an Additive," J. Chem., vol. 2015, pp. 1-8, 2015.

[16] S. Acevedo et al., "Molecular weight of petroleum asphaltenes: A comparison between mass spectrometry and vapor pressure osmometry," Energy and Fuels, vol. 19, no. 4, pp. 1548-1560, 2005.

[17] J. F. McKAY, P. J. AMEND, T. E. COGSWELL, P. M. HARNSBERGER, R. B. ERICKSON, and D. R. LATHAM, "Petroleum Asphaltenes: Chemistry and Composition," pp. 128-142, 2009. 
[18] M. R. Gray, Upgrading Oilsands Bitumen and Heavy Oil. The University of Alberta Press, 2015.

[19] R. I. Rueda-Velásquez, H. Freund, K. Qian, W. N. Olmstead, and M. R. Gray, "Characterization of asphaltene building blocks by cracking under favorable hydrogenation conditions," Energy and Fuels, vol. 27, no. 4, pp. 1817-1829, 2013.

[20] A. M. Elsharkawy, T. A. Al-sahhaf, M. A. Fahim, H. W. Yarranton, and P. Engineering, "Characterization of Asphaltenes and Resins Separated from Waterin-Crude Oil Emulsions formed in Kuwaiti Oil Fields," Chem. Eng., no. 403, 2005. [21] V. Calemma, P. Iwanski, M. Nali, R. Scotti, and L. Montanari, "Structural Characterization of Asphaltenes of Different Origins," Energy and Fuels, vol. 9, no. 2, pp. 225-230, 1995.

[22] A. M. Elbaz, A. Gani, N. Hourani, A. H. Emwas, S. M. Sarathy, and W. L. Roberts, "TG/DTG, FT-ICR Mass Spectrometry, and NMR Spectroscopy Study of Heavy Fuel Oil," Energy and Fuels, vol. 29, no. 12, pp. 7825-7835, 2015.

[23] J. J. Verstraete, P. Schnongs, H. Dulot, and D. Hudebine, "Molecular reconstruction of heavy petroleum residue fractions," Chem. Eng. Sci., vol. 65, no. 1, pp. 304-312, 2010.

[24] I. A. Wiehe, "A solvent-resid diagram for tracking resid conversion," pp. 530-536, 1992.

[25] T. Ren, Y. Wang, and G. Zhang, "Influence of the properties of resins on the interactions between asphaltenes and resins," Pet. Sci. Technol., vol. 35, no. 14, pp. 1481-1486, 2017. 